\title{
Supporting involvement of electric vehicles in distribution grids: Lowering the barriers
} for a proactive integration

\author{
Knezovic, Katarina; Marinelli, Mattia; Zecchino, Antonio; Andersen, Peter Bach; Træholt, Chresten
}

Published in:

Energy

Link to article, DOI:

10.1016/j.energy.2017.06.075

Publication date:

2017

Document Version

Peer reviewed version

Link back to DTU Orbit

Citation (APA):

Knezovic, K., Marinelli, M., Zecchino, A., Andersen, P. B., \& Træholt, C. (2017). Supporting involvement of electric vehicles in distribution grids: Lowering the barriers for a proactive integration. Energy, 134, 458-468. https://doi.org/10.1016/j.energy.2017.06.075

\section{General rights}

Copyright and moral rights for the publications made accessible in the public portal are retained by the authors and/or other copyright owners and it is a condition of accessing publications that users recognise and abide by the legal requirements associated with these rights.

- Users may download and print one copy of any publication from the public portal for the purpose of private study or research.

- You may not further distribute the material or use it for any profit-making activity or commercial gain

- You may freely distribute the URL identifying the publication in the public portal 


\title{
Supporting involvement of electric vehicles in distribution grids: Lowering the barriers for a proactive integration
}

\author{
Katarina Knezovića ${ }^{\mathrm{a}}$ Mattia Marinelli*a, Antonio Zecchino ${ }^{\mathrm{a}}$, Peter Bach Andersen ${ }^{\mathrm{a}}$, \\ Chresten Traeholt ${ }^{\mathrm{a}}$ \\ ${ }^{a}$ Center for Electric Power and Energy, Department of Electrical Engineering, Technical University of \\ Denmark, Denmark
}

\begin{abstract}
Increasing environmental concerns are driving an evolution of the energy system in which electric vehicles (EVs) play an important role. Still, as the EV number increases, the adverse impact of charging is observed more widely, especially at the low-voltage level where high EV concentrations cause various detrimental effects due to the coincidence between EV charging and residential peak load. However, if managed properly, EVs become flexible resources which can improve the system operation, making them an attractive asset for the distribution system operator. With the recent technology development, new forms of local EV support can be developed, provided that an appropriate regulatory framework is established. Whereas the technical value of such EV distribution grid services has already been proven, integrating them into the European regulatory context is not straightforward. In the context where active distribution grid management schemes are still to be developed, it is important to recognise the barriers for active EV involvement in the early stage of the development. This manuscript focuses on identifying these barriers from a technology and infrastructure perspective as well as from the regulatory and market aspect. Various policy recommendations are provided for the stakeholders involved in the EV value chain.
\end{abstract}

Keywords: distribution grid, electric vehicle, flexibility service, regulatory barriers

\footnotetext{
*Corresponding author

Email addresses: kknez@elektro.dtu.dk (Katarina Knezović), matm@elektro.dtu.dk (Mattia Marinelli*), antozec@elektro.dtu.dk (Antonio Zecchino), pba@elektro.dtu.dk (Peter Bach Andersen), ctr@elektro.dtu.dk (Chresten Traeholt)
} 


\section{Introduction}

Increasing environmental concerns are driving the evolution of the energy system in which the electrification of the transport sector is considered a crucial element in achieving the set sustainability goals. Successful electric vehicle (EV) introduction allows the reduction of $\mathrm{CO}_{2}$ emissions, but also represents a challenge of daunting proportions for the power system. As the number of EVs increases, the impact of uncontrolled charging is observed more widely, especially at the distribution level where high EV concentrations cause various detrimental effects due to the to the coincidence between the EV charging and the peak residential consumption. It is generally agreed upon that, if not managed properly, EVs will cause challenges that may lead to grid over-investment in order to cope with the extreme operating conditions $[1,2,3]$. However, EVs should not be considered merely as passive loads as they hold potential for providing services beyond transportation due to their defining characteristics: they are a considerably large load compared to other conventional residential loads, they are idle more than $90 \%$ of the day with a high degree of flexibility, and they are a quick-response unit with an attached storage and potential capabilities for bi-directional power flow [4]. If managed properly, EVs become resources which can be used to enhance the system operation by providing flexibility, making them an attractive asset for the distribution system operator (DSO) [5, 6].

Nevertheless, procuring EV flexibility at the distribution level is far away from being realised despite the technical value shown in various pilot projects and numerous theoretical studies. Indeed, exploiting EV flexibility to support the distribution system operation has been negligible up to now as the organisational and regulatory aspect remain unclear for such distribution grid services. Hence, it is becoming increasingly important to systematically and thoroughly investigate the requirements for enabling active EV participation in distribution grids both from the technical perspective and the regulatory aspect. The regulatory requirements for active participation of various demand response units have been tackled in reports by relevant regulatory and industrial institutions, such as the Smart Energy Demand Coalition (SEDC) [7], Council of European Energy Regulators (CEER) [8], and The Union 
of the Electricity Industry (Eurelectric) $[9,10,11]$. Although the authors are aware of works on reviewing EV smart charging algorithms at the distribution level $[5,12,13]$, the aim of this manuscript is not to review the possible control strategies. On the contrary, the aim is to provide a comprehensive understanding about the barriers which prevent commercial actors, e.g., EV aggregators to exploit such smart charging algorithms and make EVs an integral part of active distribution grids. The focus is put on reviewing the existing literature and the distribution sector status in several European countries in order to identify barriers for active EV involvement, and provide recommendations for overcoming them. The main contributions of the paper are as follows:

- Definition of an EV flexibility service with specific technical attributes which must be addressed when procuring flexibility products as well as a classification of prominent services EVs can provide to the DSO to optimise grid operation and defer grid reinforcement.

- Identification of main technology and infrastructure related barriers as well as regulatory and market related barriers that potentially obstruct successful EV integration and deployment of distribution grid services.

- Proposal of series of recommendations for overcoming the recognised barriers with a respective roadmap for supporting active EV involvement in the distribution grids.

The remainder of this manuscript is organised as follows. Section 2 provides a conceptual basis including an overview of historical distribution grid operation with the emerging changes, the definition of an EV flexibility service and the introduction of prominent EV distribution grid services. Further, in Section 3, the main barriers for active EV involvement at the distribution level are analysed. Finally, the general policy recommendations are given in Section 4 followed by a conclusion in Section 5 . 


\section{Value of EV flexibility at the distribution level}

Before describing the potential value of EV flexibility, it is necessary to outline the historical grid operation and the main concerns of the respective distribution grid operator. Then, the emerging changes in the electric power system and the importance of EV flexibility can be presented in the relevant context.

\subsection{Historical distribution grid operation and emerging changes}

DSO is the entity concerned about the efficient and reliable electric power delivery to end customers whose main tasks include maintaining the distribution network and ensuring the power quality according to international and national regulations. Whereas, in Europe, the transmission system operator is usually unique for the whole transmission system of a country, the distribution sector is characterised by high diversity of DSOs [14, 6]. However, essentially everywhere, DSOs have historically operated grids with radial topology and unidirectional flows, where consumption has been largely inflexible, so grid security issues were dealt with by planning and network development methods [15]. As a matter of fact, DSO activities are mainly focused on long term planning and design rather than on real-time operation. The distribution business is generally regulated as a natural monopoly, and DSOs have a strong incentive in promoting grid reinforcement for solving management issues as they are directly remunerated for the reinforcement expenditures.

In this context, DSOs focus on solving grid contingencies, namely overloading and voltage issues. In Europe, responsible DSO must ensure that its distribution feeders are operated within the suitable voltage range according to the European standard EN 50160 [16]. In addition to voltage regulation, DSOs are mainly dealing with congestion issues as component overloading inevitably results in shorter life expectancy. Nowadays, DSOs mainly perform distribution grid regulation by adding capacitor banks, installing transformers with an onload automatic tap adjustment or reinforcing the grid, which can be rather costly, as shown in Table 1. This traditional DSO methodology is called the "fit-and-forget" approach.

With increasing DER penetration, the reliability and the economical operation of the power system become non-trivial since new resources impose additional constraints and 
Table 1: Assets cost, adapted from [1, 17].

\begin{tabular}{cc}
\hline \hline Component & Estimated cost \\
\hline MV over-head lines/cables & $100-200 \mathrm{k} € / \mathrm{km}$ \\
\hline LV cables & $70-100 \mathrm{k} € / \mathrm{km}$ \\
\hline LV over-head lines & $30-65 \mathrm{k} € / \mathrm{km}$ \\
\hline ground-mounted MV $/ \mathrm{LV}$ transformer & $14-35 \mathrm{k} €$ \\
\hline pole-mounted MV $/ \mathrm{LV}$ transformer & $5 \mathrm{k} €$ \\
\hline $\mathrm{HV} / \mathrm{MV}$ transformer & $1700-5200 \mathrm{k} €$ \\
\hline \hline
\end{tabular}

challenges to the system such as unpredictability, intermittency and bi-directional flows. In addition, considering the adverse effects of uncontrolled EV charging, the integration of high EV numbers cannot be done by the traditional "fit-and-forget" approach as great grid reinforcement would be needed, resulting in an overall high cost for the society. With the liberalization of the electricity industry and the recent technological improvements, a new kind of DSO is needed [18]. In order to efficiently solve the operational challenges and fulfil the core responsibilities, DSOs could exploit flexibility for achieving the technical objectives linked to their physical assets and grid constraints. The new design could also include a market mechanism at the distribution level in which available, feasible and cost-effective solutions become part of any distribution system planning efforts. This new methodology of investments, management and remuneration of decentralized flexibility resources, including EVs, is called the "proactive distribution grid operation".

\subsection{The definition of an EV flexibility service}

In general, EV flexibility service can be defined as a power adjustment maintained from a particular moment for a certain duration at a specific location. Despite the fact that flexibility services can be provided by the individual EV, some can have a significant impact only if provided by a large fleet. In order to make such management possible, the existence of a dedicated entity is required, which is often called EV aggregator and typically acts as the middleman among EV owners and power system stakeholders [19, 20]. Regardless if the required flexibility is provided by an individual $\mathrm{EV}$ or a pool of aggregated EVs, the flexibility service is characterised by five theoretical attributes, as seen in Figure 1a as well as by five practical attributes which arise due to resource imperfections, as shown in Figure 1b. 
(a)

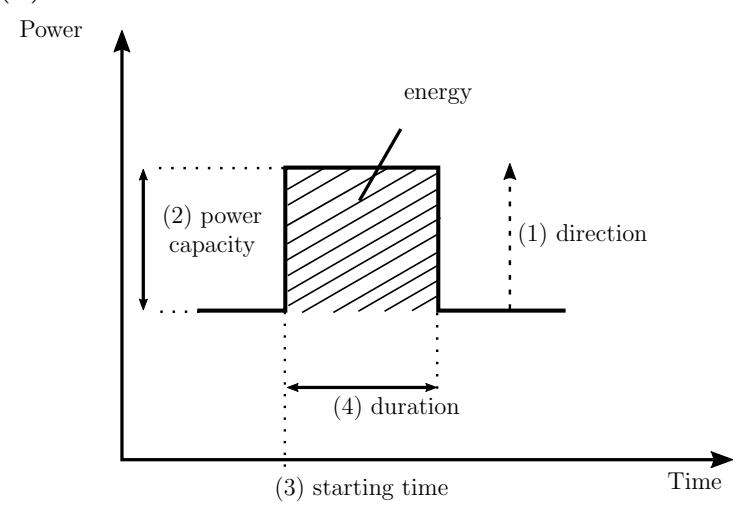

(b)

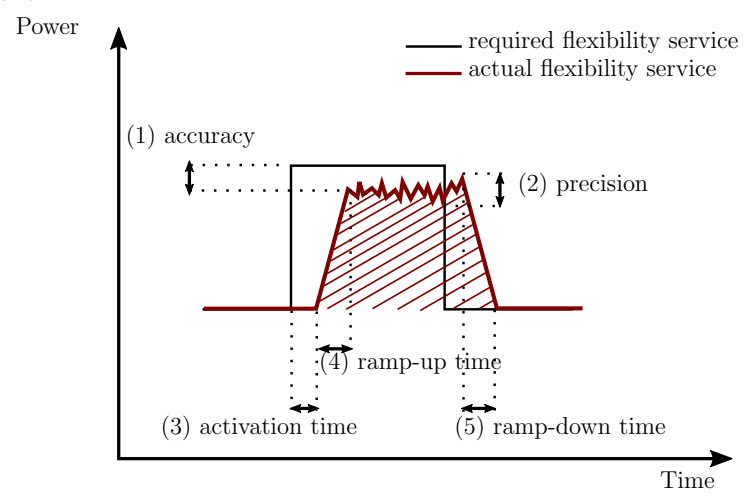

Figure 1: (a) Theoretical attributes of a flexibility service (excluding the location), and (b) practical attributes of a flexibility service.

The theoretical attributes are:

- Direction: The information if an EV can provide only unidirectional or bidirectional power flow must be known as well as the information on reactive power capabilities. These properties are obtained through contracts with the EV owners. The DSO requests and the EV offers a flexibility service of a certain power direction.

- Power capacity: Limitations on available capabilities are required such as the nominal rating of the charging equipment and the active/reactive power capability. The required/offered power capacity must be defined for each flexibility request/offer.

- Starting time: The DSO requests and the EV offers a flexibility service from a particular starting time which defines the temporal characteristics of the service.

- Duration: The period within which flexibility is acquired must be defined in the flexibility request/offer. Then, the maximum energy which can be requested in the contracting period is implicitly contained through the required power capacity and the duration.

- Location: Location of a flexible EV can be defined either as the node of coupling or as the corresponding superior substation depending on the required service. For 
example, exact EV location is of little importance if the EV is providing congestion prevention as long as it is supplied through the congested transformer, whereas the voltage regulation service is highly dependent on the point of common coupling.

The practical attributes are:

- Accuracy: The acceptable difference between the required and the delivered response must be defined, e.g., the acceptable response band.

- Precision: The acceptable variation of the delivered response must be defined, i.e., the amount of variation that exists in the delivered response for the same required value.

- Activation time: The period between receiving the required set-point and activating the required flexibility must be determined. More precisely, the DSO defines the maximum acceptable activation time in the flexibility request and the EV aggregator defines the maximum activation time of its resources in the flexibility offer.

- Ramp-up time: The period between activating the required flexibility and reaching the new set point which is greater than the current operating point. The acceptable upwards rate-of-change duration between the activation time and full service provision must be defined.

- Ramp-down time: The period between deactivating the required flexibility and reaching the new set point which is lower than the current operating point. The acceptable downwards rate-of-change duration for service deactivation must be defined.

\subsection{Prominent EV distribution grid services}

With respect to EV flexibility services which can be provided to the DSO, different objectives can be taken into account. One has to bear in mind how the classification described here is just one of the possible categorisations which is derived based on the literature survey and the current DSO operation. These services correspond to the DSO's needs, but may not be the exact products defined in the future. In general, EV flexibility services for 

as depicted in Figure 2.

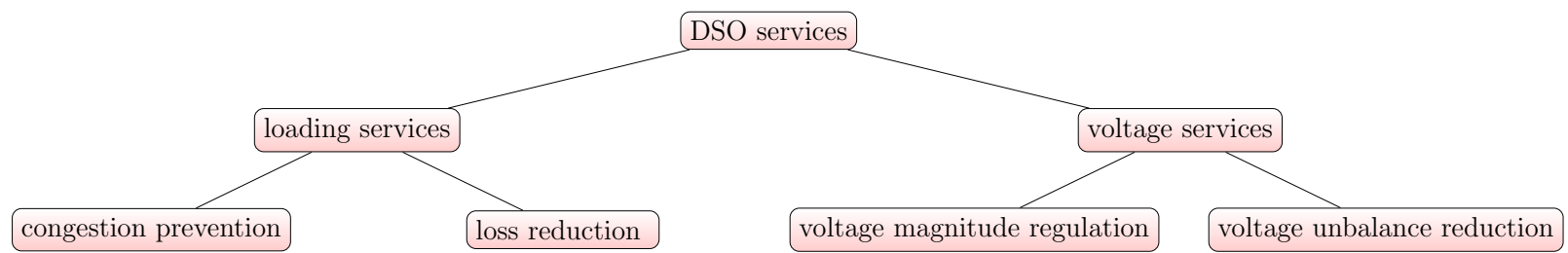

Figure 2: Classification of possible services EVs can provide to the DSO.

achieving the technical objectives can be divided in two groups depending on the targeted grid constraint, namely services for solving rated capacity issues and services for solving voltage issues. These two groups can further be split into several distribution grid services

In the EV related literature, a wide range of algorithms for achieving the set objectives can be found, both for direct load management and indirect price control schemes as well as for different control architectures. For example, controlling the adverse EV voltage effects has been investigated in $[21,22,23]$, congestion prevention methods have been studied in $[24,25,26]$, whereas the loss reduction provision has been analysed in [27, 28]. It is generally agreed that EVs can provide services to mitigate the self-inflicted adverse effects as well as to compensate for the undesirable effects of other distributed renewable resources. However, whereas the technical value to the system has been proven for different EV operational strategies, integrating EV distribution grid services into the European regulatory context is not straightforward. Therefore, it is important to assess the current status from four aspects: (1) enabling EV participation and aggregation, (2) standardised measurement, communication and verification requirements, (3) payment structures, and (4) appropriate programme requirements for distribution grid services (minimum bid, penalty for non-delivery, etc.).

\section{Barriers and challenges for proactive EV involvement at the distribution level}

In a liberalised environment, local distribution grid support can be acquired either through mandatory grid codes or through trading of flexibility services. Unless a certain EV flexibility service is made mandatory, a number of issues must be investigated by the relevant stakeholders to make it a tradable commodity. When dealing with EV flexibility 
provision for emerging DSO services, key prerequisites must be identified as guidelines for large-scale procurement, regardless if the remunerated services are obtained through bilateral contracts or a local flexibility market. Indeed, the real applicability of EV distribution grid services will highly depend on the local regulatory conditions as well as on the deployed infrastructure. Hence, it is important to analyse the techno-institutional and the economic layers [29], with emphasis on recognising barriers for active EV involvement and providing recommendations for overcoming them. These barriers and challenges can be divided in two categories: the technology and infrastructure related ones, and the policy and market related ones.

\subsection{Technology and infrastructure related barriers}

This section is concerned with the main barriers for an efficient utilisation of EV flexibility at the distribution level which are related to technology and infrastructure, and can be observed across Europe. Special attention is put on practical attributes of EV flexibility services, grid observability and smart metering, deployment of EV supply equipment (EVSE), and the related standardisation support.

\subsubsection{Assessing practical attributes of EV flexibility}

If EVs are to be treated as "black boxes" when providing flexibility services, their internal parameters must be carefully addressed in order to provide both the DSO and the EV aggregator with the knowledge of the EV technical capabilities and the means for compensating the imperfections. It is clear that the practical attributes of EV flexibility services, such as the accuracy, the precision and the response time, must be thoroughly investigated for a vast amount of EV brands and models in order to test their ability to comply with flexibility service requirements. Yet, the vast majority of the EV related literature remains on simulation studies, whereas the experimental testing has widely been neglected, making it hard to evaluate the true value of EV flexibility.

In $[30,31,32]$, the authors focused on validating the technical feasibility of current seriesproduced EVs to provide different flexibility services through laboratory and field trials. The results provided various indications of the contemporary EV capabilities, but they are far 
away from being exhaustive. More specifically, the conducted analyses showed that EVs have a fast response within several seconds, but there is a significant difference in response accuracy based on the external conditions such as the ambient temperature, which arose as a topic of concern [32]. Moreover, the conducted experiments were done with a single EV model, so other series-produced EVs might not have the same response delays and inaccuracies as the ones obtained in these studies. The clear lack of experimental data for assessing the reliability of series-produced EVs to provide distribution grid services as well as evaluating their contemporary capabilities under various external conditions is seen as a major barrier.

\subsubsection{Grid observability and smart metering}

It is widely acknowledged that the mass roll-out of smart meters is the main facilitator for enabling flexibility procurement since the accurate measurement of consumption patterns is crucial for an effective billing [33]. Measurements from the bottom of the distribution grid could provide the DSO with more knowledge about the respective grid, making it capable of judging if flexibility procurement is needed or grid reinforcement is inevitable. The European Electricity Directive [34] requires the member states to ensure that at least $80 \%$ of consumers are equipped with smart meters by 2020 unless the conducted costbenefit analysis provides indications that the roll-out volume should be smaller. As seen in Table 2, several European countries have plans for a wide-scale roll-out of smart meters supported by the national regulatory framework. Yet, there is still a relatively large share of countries which have not started the deployment due to negative or inconclusive results of the cost-benefit analysis. In majority of the countries where smart meters are deployed, all units are certified and installed by the DSO, who is also responsible for data collection and management. Regardless if the DSO or an independent third party is deploying the meters, it is of particular importance to clearly define the requirements on the specific measurement parameters such as the sampling rate, which must be chosen as a trade-off between the information speed on one hand, and the installation and data management cost on the other. According to the European Commission's recommendation [35], smart meter 
Table 2: Current status for several European countries in case of smart metering infrastructure [36, 37].

\begin{tabular}{|c|c|c|c|}
\hline Country & Wide-scale roll-out by $2020^{a}$ & Sampling rate & Data management responsible \\
\hline Belgium & o & $\bigcirc$ & DSO \\
\hline Denmark & - & $15 \mathrm{~min} / 1 \mathrm{~h}^{\mathrm{b}}$ & DSO \\
\hline France & - & $30 \mathrm{~min}$ & DSO \\
\hline Germany & 0 & $15 \mathrm{~min}$ & meter operator/DSO \\
\hline Ireland & - & $30 \min$ & DSO \\
\hline Italy & - & $10 \mathrm{~min}$ & $\mathrm{DSO}$ \\
\hline Netherlands & - & $15 \mathrm{~min}$ & $\mathrm{DSO}$ \\
\hline Spain & - & 0 & DSO \\
\hline UK & $\bullet$ & $15 \mathrm{~min}$ & supplier \\
\hline
\end{tabular}

a $\bigcirc$ criteria is not fulfilled, $\odot=$ criteria is fulfilled to some extent, $\bullet=$ criteria is fulfilled

b $1 \mathrm{~h}$ for smart meters installed until 2011, 15 min for the meters installed after 2011

functionalities should include remote reading with two-way communication and a sampling rate not greater than 15-min. Yet, there is no international standards which would ensure these functionalities, so the status across Europe considerably varies.

The lack of homogeneous and standardised functionalities among smart meters prevents more sophisticated ways of flexibility procurement and is observed to be one of the major barriers. The same barrier applies to advanced metering infrastructure which must be available for individual EVs to allow verification of the flexibility delivery.

\subsubsection{EV supply equipment}

All users should have a non-discriminatory access to electricity network [38] and the same principle applies for the EV connection. Since the EV presence is relatively small in most of the European countries, national grid codes do not include any connection requirements considering the respective EV supply equipment as DSOs have not yet encountered any major challenges. However, as EV number increases, EV charging will have a significant influence on the distribution system operation and the dedicated connection requirements will be needed, similarly to the mass adoption of PV installations which resulted in revisiting the grid connection rules $[39,40]$. The most important requirements concerned the reactive power compensation, so it is expected that such will be necessary for EVs as well [23, 41].

Further on, the use of EVSEs with sufficient computational and communication capabilities is the key for enabling advanced flexibility services as it allows controlled EV charging, 
either autonomously or in a coordinated fashion. Whereas there is already commercially available equipment which allows the controlled EV charging, including the communication and the computational capabilities in the contemporary EVSEs is not a common practice as it imposes an additional cost. If such capabilities would be included from the beginning of the infrastructure roll-out, the additional cost of retrofitting the older EVSEs once EV smart charging becomes a common practice would be avoided. Another important aspect is EV identification since a standardised way of assigning a unique ID number to the individual EVSE, or alternatively to the EV, must be defined to ensure that the proper user is procured and remunerated for the delivered flexibility. Moreover, the basic EV information, such as the plug-in time, the maximum battery capacity and the initial SOC when plugged-in, should be recorded at the EVSE level by the respective measurement equipment. These information should also be made accessible by the EV manufacturers, which is, e.g., currently not the case for the SOC data. Naturally, user privacy must be ensured by regulations, so that all collected data are treated as confidential and kept private. Finally, EV users must be properly informed and provided with the tools to understand the complex contracts to which they can be exposed. It is necessary to develop EV interfaces which are user-friendly and provide insight into the signed contracts as well as the scheduled EV operation. Otherwise, the user willingness to participate in flexibility schemes could be jeopardised.

\subsubsection{EV communication standards}

When talking about EV flexibility procurement, the practical implementation must guarantee interoperability between different equipment and the involved stakeholders. The mapping of the most important contemporary standards for supporting EV distribution grid services is depicted in Figure 3. Nowadays, the vast majority of contemporary EVs are compliant with IEC 61851 [42] or SAE J1772 standard [43] according to which the EV charging current can be limited between the minimum charging current of $6 \mathrm{~A}$ and the maximum one, which is the EVSE rated current (10 A, 16 A, 32 A, etc.), in discrete 1 A steps. Such capability of limiting the current is seen as the first step in enabling EV distribution grid 


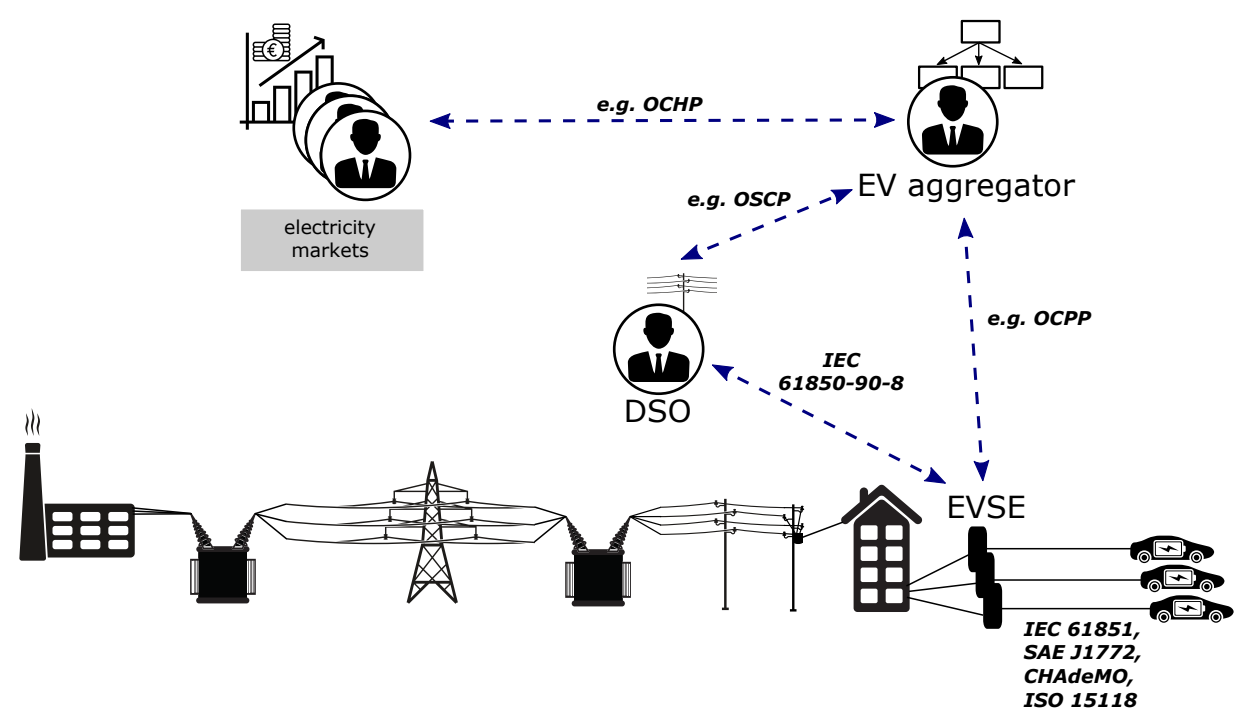

Figure 3: Relevant EV standards and protocols between power system stakeholders with respect to EV distribution grid services.

services. As opposed to the low level communication described in these standards, a newer standard ISO/IEC 15118 [44] covers information exchange between all actors involved in the electrical energy supply process to the EV, taking into account the data encryption for both confidentiality and data integrity purposes. This standard is highly relevant for EV flexibility procurement, yet it is not widely supported by the contemporary EV equipment since it is still under development. Also, one of the major drawbacks of this standard is that it does not require SOC data which is seen as a necessity for most of smart charging algorithms. The scope of IEC TR 61850-90-8 is to describe the communication link between EVSEs and the power system operator as well as to harmonize information flow models independent of the underlying hardware and software protocols. Still, the standard is expected to be included in the second edition of IEC 61850-7-420 and is not widely supported. Additionally, three open application protocols are relevant for procuring EV distribution grid services due to the lack of international standards: the Open Charge Point Protocol (OCPP) [45] for the communication between the EVSE and the EV aggregator; the Open Clearing House Protocol (OCHP) [46] for the communication between the EV service provider and the clearing house system; and the Open Smart Charging Protocol (OSCP) [47] for communication between the EV aggregator and the DSO. 
As EV flexibility provision is not a common practice, the lack of international standards for supporting it is not surprising. Still, this lack represents a major barrier for utilising the full-scale potential of EV flexibility at the distribution level.

\subsection{Regulation and market related barriers}

Since DSOs are natural monopolies, the support of the regulatory framework is essential, so identifying and overcoming the regulatory barriers is crucial to ensure that the future distribution system effectively deals with EV integration. This section focuses on identifying the policy and market related barriers with emphasis on the DSO business paradigm including the aggregation regulation and remuneration schemes as well as the potential introduction of local platforms for flexibility trading.

\subsubsection{DSO business paradigm}

Even though DSO regulatory frameworks differ from country to country, some common factors for enabling EV distribution grid services can be clearly identified. First of all, to procure any kind of flexibility, these actions must be allowed by the respective regulation, which includes the regulation for introducing independent EV aggregators as well as for DSOs contracting flexibility services. Currently, many national regulations do not explicitly allow flexibility procurement and some even forbid the aggregation, as seen in Table 3 . This major barrier must be addressed as soon as possible.

Table 3: Current status for several European countries with respect to DSO regulation [7, 37, 11, 48].

\begin{tabular}{|c|c|c|c|c|}
\hline Country & $\begin{array}{c}\text { Aggregation enabled } \\
\text { by regulation a }\end{array}$ & $\begin{array}{l}\text { Network tariff } \\
\text { structure }^{b}\end{array}$ & $\begin{array}{c}\text { DSO regulatory } \\
\text { period (years) }\end{array}$ & $\begin{array}{c}\text { Mechanisms for } \\
\text { stimulating innovation a }\end{array}$ \\
\hline Belgium & (1) & $€+€ / \mathrm{kWh}$ & 4 & 0 \\
\hline Denmark & (1) & $(€)^{\mathrm{c}}+€ / \mathrm{kWh}$ & 3 & (1) \\
\hline France & O & $€+€ / \mathrm{kW}+€ / \mathrm{kWh}$ & 4 & O \\
\hline Germany & (1) & $€+€ / \mathrm{kWh}$ & 5 & $\bigcirc$ \\
\hline Ireland & (1) & $€+€ / \mathrm{kWh}$ & 5 & (1) \\
\hline Italy & O & $€+€ / \mathrm{kW}+€ / \mathrm{kWh}$ & 4 & (1) \\
\hline Netherlands & (1) & $€+€ / \mathrm{kW}+(€ / \mathrm{kVArh})^{\mathrm{c}}$ & 3 & $\bigcirc$ \\
\hline Spain & $\bigcirc$ & $€ / \mathrm{kW}+€ / \mathrm{kWh}$ & 6 & O \\
\hline UK & (1) & $€+€ / \mathrm{kWh}$ & 8 & (1) \\
\hline
\end{tabular}


Secondly, DSOs are regulated entities which recover their cost through regulated revenues based on a cost-of-service method or an incentive-based method [15]. For both methods, DSO costs are calculated by evaluating the operational expenditures (OPEX) and the capital expenditures (CAPEX) which are then included in the regulatory formula for the chosen remuneration approach. Incentive regulation is a common practice across Europe after deregulation of the electricity sector [48]. In such a scheme, the regulator sets the allowed yearly revenues for the regulatory period, and the DSO can gain an extra profit by decoupling the costs from the revenue and increasing the efficiency. However, in practice, it is difficult to regulate the long technical and economic lifetime of grid components, so regulators exclude CAPEX from the efficiency requirements and remunerate the actual cost of grid reinforcement, which effectively discourages DSOs from active grid management.

Bearing this barrier in mind, it is necessary to revise the current incentives for performing the traditional DSO tasks, including the remuneration and tariff structures [49, 11]. Ideally, the regulation should provide explicit support via incentives for acquiring flexibility services in addition to incentives for reducing the cost both for the capital and the operational expenditures. Moreover, the regulated electricity tariffs must be designed in order to ensure the full cost-recovery for the DSO's allowed expenses while encouraging a more efficient grid use. As network upgrades will still be needed, the electricity tariff should include at least two components: a capacity $(€ / \mathrm{kW})$ and an energy component $(€ / \mathrm{kWh})$, which is currently not the case in many European countries, as seen from Table 3. The capacity component would cover the necessary grid reinforcement cost and discourage high instantaneous power consumption, whereas the energy component could vary to reflect the local network conditions. Another aspect which must be taken into account is the regulatory period which often does not incentivise the long-term innovation. As shown in Table 3, the regulatory periods usually last for 4 or 5 years which is too short to see major efficiency improvements from EV flexibility. Additionally, in most of the countries, there is no direct mechanisms to stimulate innovation in the distribution networks. 
A possible organisation of such a framework is given in Figure 4.

\subsubsection{Local flexibility trading}

Unless certain EV distribution grid service is made mandatory through grid codes, it will be treated as a commodity which can be either directly invoked by the DSO for a fixed price or traded on the market. As pointed out in [50], it is still unclear who should initiate the development of local DSO markets or if the trading should be on bilateral basis due to locational restrictions. However, it is mainly agreed that a dedicated flexibility platform is needed to invoke flexibility trading [10], as via such interface DSOs could require and service providers, including EV aggregators, could offer flexibility. The open platform would enable trading of flexibility products through different markets with their own rules, or could be used for contracting services on bilateral basis if local flexibility markets are not established.

(a)

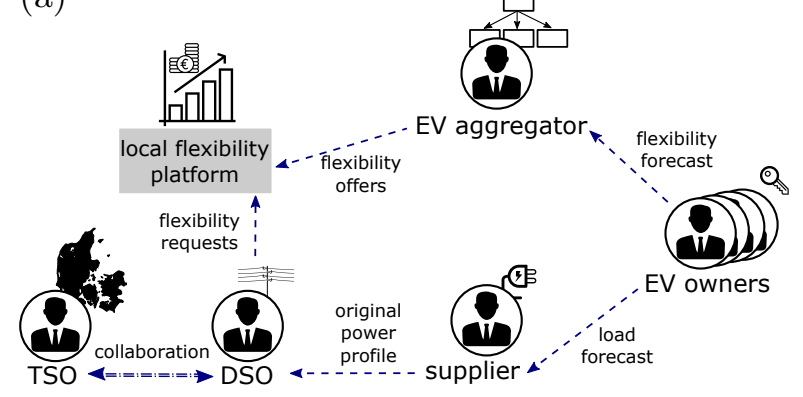

(b)

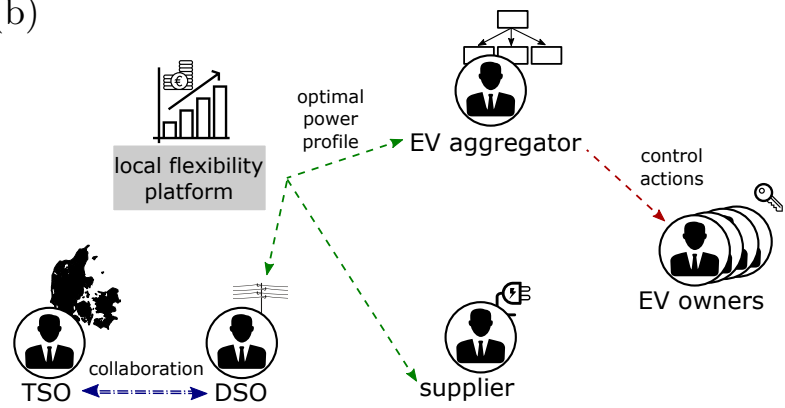

Figure 4: Possible local flexibility framework for the day-ahead trading of EV distribution grid services: (a) before, and (b) after the clearing process. The TSO-DSO collaboration is indicated without a detailed elaboration, as the focus is put on the local level. Based on [51, 52].

Trading EV flexibility at the distribution level is nowadays non-existent in all European countries, and the lack of dedicated platforms is identified as a major barrier. Without defining a number of regulatory aspects to establish such platforms, potential EV flexibility will remain unused. These aspects include:

- Flexibility platform administration and operation: It is conceptually possible to have separate entities for the distribution system operation and the distribution system flexibility operation. Some claim that assigning a dual role to the future DSO is more beneficial as the DSO is aware of the grid status and operational conditions [53]. However, this can also lead to market manipulations depending on the regulatory 
environment, so an independent entity may be needed. Regardless, the flexibility operator must manage and operate the flexibility platform by accumulating the bids and obtaining the optimal EV schedules.

- Independence and fair access: Flexibility operator must be independent of any participant or EV owner to operate flexibility trading in a fair and an impartial manner, and it should not own any flexibility assets in the corresponding distribution area to avoid conflict of interest. Regulations are required to ensure open and fair platform access for all interested participants.

- Transparency: Participants must have access to financial information such as the cleared prices, whereas the bidding process, if existing, should be blind. The flexibility framework must be transparent in terms of data exchange among different parties, rules on the clearing process, operating costs and system operation procedures. Clarity is needed on criteria how to become a participant with the corresponding prequalification process, respective rights and obligations as well as criteria for terminating the participation.

- Flexibility products: Clear and generic flexibility products must be defined with clear conditions for procurement and defined requirements including the aforementioned theoretical and practical attributes (response time, accuracy, power capacity, duration, etc.). Contractual arrangements should be simple, transparent and fair to allow all willing EV owners to participate in such schemes.

- Minimum bid: Power consumption at the distribution level is of much lower values than at the transmission level, so even one EV can be a valuable asset for a distribution feeder. If flexibility trading is introduced, lowering the requirements for minimum participation would allow easier entry of many players to the local flexibility platform.

- Settlement period: The settlement frequency must correspond to the measurement interval, i.e., the settlement period should not be lower than the data sampling rate. 
From the DSO perspective, sampling rates on second basis are not a necessity, but such could be of additional value if EVs were to provide services to the TSO as well.

- Consumption baseline: Flexibility only exists because we can estimate what would the load profile look like if flexibility was not activated, but after all, only the actual load profile can be measured and the unperturbed one never existed. If a common baseline is not accepted by all involved participants, many settlement disputes will arise.

- Flexibility price: The price for each flexibility product should be determined and transparently communicated in advance. However, how it should be defined is not straightforward as it is not easy to assess the value of demand shifting and potential impact on the user comfort, making it difficult to assign a monetary value for providing flexibility. In any case, the settled price must be lower than the cost of grid reinforcement. The maximum price $C_{\max }$ that the DSO is willing to pay for reserving the flexibility service can be defined as follows [54]:

$$
C_{\text {max }}=\left(C_{\text {reinforcement }}-N_{\text {activation }} \cdot \lambda_{\text {activation }}-C_{\text {transaction }}\right) \cdot(1-u)
$$

where $C_{\text {reinforcement }}$ is the present value of the deferred cost for grid reinforcement, $N_{\text {activation }}$ is the expected number of service activations, $\lambda_{\text {activation }}$ is the activation price determined in the contract, $C_{\text {transaction }}$ is the cost of transaction and $u$ is the uncertainty premium which reflects the DSO's risk preferences. The activation price $\lambda_{\text {activation }}$ is dependent both on the capacity and the duration of the required service and reflects the aggregator's operational cost which is determined for each flexibility offer. The uncertainty premium $u$ directly rewards the more reliable resources, since the DSO can decrease the premium for the resources which are considered to be less risky. Moreover, as flexibility trading develops and many participant get involved, the transactions costs are expected to decrease.

It is important to note how this list is not exhaustive and many other aspects must be addressed as well. For example, it is important to define how local flexibility platforms 
would interact with the wholesale electricity market and the parties involved in those trading processes.

\subsubsection{Collaboration between the TSO and the DSO}

When procuring EV distribution grid service, the interaction between the DSO and the TSO must be ensured, particularly if services at the distribution level inadequately interact with the transmission system needs and trigger the need for system-wide services. The coordination of resources for both the DSO and the TSO purposes is needed, and procurement of distribution grid services needs to take into account the effects on the TSO operation. Nowadays, the interaction between the TSO and the DSO is limited, and for such reasons there is an increasing attention put on improving the TSO-DSO relationship $[55,56,57]$. The regulations must ensure that data sharing is free of charge for all eligible players and that the processes for data exchange are defined with clear responsibility for data management.

\section{Policy recommendations}

Based on the current regulatory and infrastructure status across the European distribution sector as well as the previously identified barriers, a series of recommendations is provided as guidelines for transitioning to a future flexible distribution system where EVs become proactive participants at the distribution level. These recommendations are divided in several categories depending on the targeted aspect, as presented in Table 4. Additionally, the phases for the listed recommendations as well as the intermediate steps needed for fulfilling them are presented via the roadmap depicted in Figure 5.

With respect to smart-metering and EV metering infrastructure, it is recognised that international standards are needed to define basic smart-meter functionalities and ensure interoperability among all participants. From the EV integration perspective, the sampling rate should correspond to the settlement period, which should be as short as possible and the authors believe it should not be higher than 5 minutes. This is seen as a psychological limit which would not impose a high inconvenience for the owner in case the EV is unavailable 
during the contracted period, so the user has to wait until flexibility provision is terminated without incurring the penalties for non-delivery. The settlement period above 5 minutes may discourage the user to participate in flexibility trading as it can influence his comfort. This recommendation is also aligned with [58] where a 5-min sampling rate has been recognised as a trade-off between the related metering and communication cost, and the system performance. Regardless of the chosen sampling rate, international standards are needed in the near future to ensure that the rolled-out infrastructure is interoperable, and to avoid cost for retrofitting the unsuitable equipment once flexibility trading becomes well-established. Moreover, in order to reduce the overall system complexity and consequently the cost, the meters installed in the EVSEs should also serve for flexibility settlement purposes. However, in order to make such a system viable, clear verification and pre-qualification protocols must be defined for the EVSE measurement equipment in addition to the responsible parties for carrying out the validation and the data management. If EVs are providing services for the TSO, the same measurement equipment can also be used to validate the services provided to the DSOs since one can assume that if EV satisfies frequency control requirements, it would also satisfy the ones for DSO services as overloading and voltage issues are of much slower nature.

One of the main recommendations with respect to the contemporary EV technology is establishing standardised tests for evaluating the internal EV parameters, including the accuracy and the response time. This would enable benchmarking various vehicles to each other and encourage EV manufacturers to improve the grid integration performance. The collected data could also be used for further theoretical research studies such as valuing EV flexibility or system-identification for establishing dynamic models of various EV models, which is of particular value for studying flexibility aggregation of numerous different EVs. Moreover, such standardised tests could also be used for the pre-qualification process to ensure that EVs are capable of providing the specified flexibility service.

In order to make EV distribution grid services possible, the deployment of infrastructure with embedded intelligence should be supported and promoted via standards and regulations in the near-future. This includes harmonisation of communication standards and protocols 
between all actors participating in flexibility procurement to ensure interoperability between various equipment and actors. Moreover, the standards should explicitly require basic EV information such as SOC data which is currently not available in many contemporary EVs. Such data becomes essential if advanced smart charging strategies are to be implemented, and is also necessary for defining the common EV baseline. More precisely, the common baseline can be constructed more easily for EVs than for other flexible resources by estimating the load demand if uncontrolled charging is applied, i.e., as the case where EV charges at the maximum rate from the plug-in time until it is completely full. For this, three parameters should be known in addition to the maximum battery capacity: the maximum charging power, the recorded initial SOC and the recorded plug-in time. Therefore, international standards must ensure that such data is available and accessible by the aggregator.

Based on the current status across Europe, regulatory barriers are observed to be a greater challenge than the technology ones, so a number of recommendations is given. First of all, it is recommended that respective regulations allow aggregation and procurement of EV flexibility services. Even if regulations do not encourage flexibility procurement, they must be revised in order to explicitly allow it. In such way, the DSO can decide to directly invoke EV flexibility for a fixed price if it assesses it to be the most cost-efficient solution. Secondly, new regulations are needed to impose transparent service remuneration of all current DSOs services. With this transparency effort, economic calculations can be performed to compare the efficiency between the "fit-and-forget" approach and "proactive solutions", and provide the basis for calculating the flexibility price and introducing local flexibility trading platforms. Thirdly, the electricity tariffs should be revised to include both a capacity and an energy component. Such tariff would encourage EV user participation in flexibility schemes as the EV is a significant load compared to other residential appliances which would increase the peak power, making the users more likely to allow EV control. Another aspect which must be take into account is the regulatory period which should be prolonged with a smooth transition between the different periods, so that the regulatory uncertainty is reduced when investing in new technologies, therefore incentivising DSOs to reduce the cost in the long-run. Recently, the regulatory period in UK has been prolonged 
to 8 years in order to encourage active distribution grid management. The authors believe that such regulatory period should be taken as a minimum in other European countries as well. Additionally, innovation funding should be established to stimulate DSO active grid management by recovering the cost of research in new technologies.

The authors believe that the first step towards full-competitive local flexibility markets is introducing local flexibility platforms which are operated by the respective DSOs since they are aware of the grid status and the needed services. Via such platform, EV aggregators could offer their services, and DSOs could bilaterally contract them. After the bi-lateral flexibility procurement is well-established, local flexibility markets can be introduced with an independent third party as the flexibility operator in order to avoid potential market manipulation. In any case, clear flexibility products with the acceptable practical attributes must be defined. These products could simply reflect the services presented in Figure 2 or could be split into a finer classification if needed. For instance, DSO may be willing to pay more for a fast emergency power reduction, so such a flexibility product should be explicitly defined. It is also necessary to introduce both capacity and energy payments to encourage user participation and remunerate not only the provided service, but also the availability to provide a service. Moreover, the minimum bid requirement for flexibility trading should reflect the fact that even one EV could be a valuable resource in certain distribution feeders. Defining the minimum bid in the kilowatt range would facilitate EV distribution grid services and allow both the DSO and the EV aggregator to be more pliable in their flexibility requests and offers.

Finally, the TSO-DSO collaboration should be improved, and two possible ways for improving it are cooperation and coordination. The former implies a mutual agreement for a set of use-cases with clear roles and defined priority list between the TSO and the DSO. Cooperation is necessary to define mandatory assistance procedures and cascading principles between the operators, especially in emergency situations. The latter one relies on the flexibility platform with a proper set of market rules to avoid double bidding and coordinate the use of flexible resources on different markets, e.g., for frequency regulation and congestion management. For instance, if EV aggregators lose money when making counter- 
effective offers, they could inherently enhance the coordination. In any case, to enhance the TSO-DSO collaboration, open and interoperable standards with clear data exchange rules should be defined for interfaces in place. Moreover, if local DSO flexibility platforms are established, they must be transparent and provide the TSO with the possibility of requesting certain service deactivation, especially in emergency situations.

Table 4: Main recommendations for supporting active EV involvement in distribution grids.

\begin{tabular}{|c|c|}
\hline $\begin{array}{c}\text { Smart } \\
\text { metering }\end{array}$ & $\begin{array}{l}\text { - Wide-scale deployment of smart meters with standardised functionalities to ensure interoperability. } \\
\text { - Sampling frequency in accordance with flexibility trading settlement period (maximum 5-min). } \\
\text { - Clear pre-qualification and validation protocols. }\end{array}$ \\
\hline $\begin{array}{l}\text { EV/EVSE } \\
\text { technology }\end{array}$ & $\begin{array}{l}\text { - Define standards and regulation for deploying EVSEs with embedded intelligence. } \\
\text { - Harmonise communication protocols between the EV aggregator and other participants. } \\
\text { - Determine standardised tests for evaluating internal EV parameters (accuracy, response time, etc.). }\end{array}$ \\
\hline $\begin{array}{l}\text { DSO } \\
\text { regulation }\end{array}$ & $\begin{array}{l}\text { - Remove regulation which forbids aggregation and flexibility procurement. } \\
\text { - Incentivise long-term innovation (longer regulatory period, incentives for new technologies, etc.). } \\
\text { - Revise tariffs to include both the capacity and the energy charge. } \\
\text { - Define new DSO tasks (active grid operation and data management). } \\
\text { - Remunerate current DSO services to provide basis for comparing different solutions and estimating } \\
\text { the flexibility price. }\end{array}$ \\
\hline $\begin{array}{l}\text { Flexibility } \\
\text { trading }\end{array}$ & $\begin{array}{l}\text { - Establish an open, transparent and fair flexibility trading platform with the corresponding roles. } \\
\text { - Define clear and generic flexibility products. } \\
\text { - Define technical requirements which must be included in flexibility requests/offers (power capacity, } \\
\text { duration, direction, location, etc.). } \\
\text { - Define the minimum bid in the kilowatt range and the settlement period of maximum 5-min to } \\
\text { encourage EV owner participation. } \\
\text { - Define common EV baseline (uncontrolled charging) and the corresponding measurement methodology. } \\
\text { - Introduce capacity and energy payments, and a premium for rewarding the more reliable resources. }\end{array}$ \\
\hline $\begin{array}{c}\text { TSO-DSO } \\
\text { collaboration }\end{array}$ & $\begin{array}{l}\text { - Define standards for the interface and data exchange between the TSO and DSOs. } \\
\text { - Define clear priorities between TSO and DSOs for normal operation and emergency situations. } \\
\text { - Make local flexibility trading platform transparent to the TSO. }\end{array}$ \\
\hline Consumer & $\begin{array}{l}\text { - Define regulations to ensure data protection and allow sharing of sensitive data if EV user is willing. } \\
\text { - Develop interface for providing insight into signed contracts and EV schedules. } \\
\text { - Define standards for providing an unique ID for flexibility procurement and remuneration. }\end{array}$ \\
\hline
\end{tabular}




\begin{tabular}{|c|c|c|c|c|}
\hline & $\begin{array}{c}\text { Initial preparation } \\
\text { and viability }\end{array}$ & $\begin{array}{l}\text { Direct invoking } \\
\text { of EV flexibility }\end{array}$ & $\begin{array}{c}\text { Local EV } \\
\text { flexibility trading }\end{array}$ & $\begin{array}{l}\text { Unleashing the } \\
\text { full EV potential }\end{array}$ \\
\hline 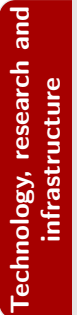 & $\begin{array}{l}\text { - Quantify and value EV } \\
\text { flexibility } \\
\text { - Increase grid observability } \\
\text { - Determine DSO grid codes } \\
\text { for EV connection } \\
\text { - Test EV capabilities of } \\
\text { providing various services } \\
\text { with small-scale trials }\end{array}$ & $\begin{array}{l}\text { - Deploy standardised EVSEs } \\
\text { - Establish direct invoking of } \\
\text { EV flexibility by the DSO for } \\
\text { a fixed price } \\
\text { - Start developing local } \\
\text { flexibility platforms with } \\
\text { large-scale field trials }\end{array}$ & $\begin{array}{l}\text { - Establish local flexibility } \\
\text { platforms with } \\
\text { semi-competitive approach } \\
\text { (flexibility procured only by } \\
\text { the DSO) } \\
\text { - Begin developing } \\
\text { full-competitive market } \\
\text { models with large-scale } \\
\text { field trials }\end{array}$ & $\begin{array}{l}\text { Establish local flexibility } \\
\text { platforms with } \\
\text { full-competitive approach } \\
\text { (flexibility procured by any } \\
\text { participant) }\end{array}$ \\
\hline 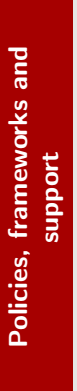 & $\begin{array}{l}\text { - Allow EV aggregation } \\
\text { - Allow DSOs to procure } \\
\text { flexibility } \\
\text { - Standardise EV metering } \\
\text { functionalities } \\
\text { - Standardise EVSE } \\
\text { communication and } \\
\text { control capabilities } \\
\text { - Remunerate DSO services }\end{array}$ & $\begin{array}{l}\text { Review DSO business } \\
\text { paradigm (new roles, new } \\
\text { electricity tariffs, flexibility } \\
\text { incentives, ...) } \\
\text { - Define clear flexibility } \\
\text { products } \\
\text { - Determine requirements } \\
\text { for flexibility trading } \\
\text { (minimum bid, settlement } \\
\text { period, ...) } \\
\text { - Standardize data exchange } \\
\text { among all parties }\end{array}$ & $\begin{array}{l}\text { - Define TSO-DSO priorities } \\
\text { and interaction for normal } \\
\text { and emergency situations } \\
\text { - Clarify jurisdiction of } \\
\text { different parties } \\
\text { - Establish market power } \\
\text { prevention mechanisms }\end{array}$ & $\begin{array}{l}\text { - Integrate local flexibility } \\
\text { markets with the wholesale } \\
\text { market } \\
\text { - Continuous revision and } \\
\text { improvement of flexibility } \\
\text { framework based on } \\
\text { lessons learned }\end{array}$ \\
\hline
\end{tabular}

Figure 5: Roadmap with key recommendations for supporting active EV involvement in distribution grids.

\section{Conclusion}

Enabling EV distribution grid services requires a coordinated participation of the full electricity value chain, but most European countries still suffer from a critical gap between the political sustainability plans and the implemented regulatory frameworks.

This paper investigated and defined the EV flexibility service, highlighting the prominent ones that could be provided to distribution system operators. In addition, it assessed the technical and the non-technical prerequisites for enabling EV flexibility procurement at the distribution level. It was observed that the identified regulatory and policy barriers present a greater challenge than the technology and infrastructure due to large diversity of distribution systems and respective regulatory frameworks across Europe. Based on the identified barriers from the technology and infrastructure aspect as well as from the policy and market perspective, a set of policy recommendations was provided for supporting the proactive EV involvement in the energy system. Since the transition to such a proactive system should be evolutionary, the phases for the listed recommendations as well as the 
intermediate steps needed for fulfilling them were presented via a roadmap.

One must bear in mind that the provided recommendations are not exhaustive. Due to system complexity and diversity across different European countries, other non-listed organisational and regulatory barriers arise both on the pan-European level and on the individual country basis. However, without addressing the listed recommendations, it will not be possible to unleash the full potential of procuring EV flexibility for distribution grid services. Moreover, political interference creates regulatory uncertainty and unique local environment may detrimentally affect the regulatory stability. Periodically comparing and contrasting various regulations across Europe is a useful source for identifying the barriers and the best-case solutions, and should become a common practice for all stakeholders involved in the EV value chain. Only then could the regulations be properly revised to ensure the technical and economic competitiveness of EVs providing distribution grid services.

\section{Acknowledgement}

This work is supported by the Danish research projects "NIKOLA - Intelligent Electric Vehicle Integration" under ForskEL kontrakt nr. 2013-1-12088, and "Parker" under ForskEL kontrakt nr. 2016-1-12410.

\section{References}

[1] L. Pieltain Fernandez, T. Gomez San Roman, R. Cossent, C. Mateo Domingo, P. Frias, Assessment of the impact of plug-in electric vehicles on distribution networks, IEEE Transactions on Power Systems 26 (1) (2011) 206-213.

[2] K. Clement-Nyns, E. Haesen, J. Driesen, The impact of charging plug-in hybrid electric vehicles on a residential distribution grid, IEEE Transactions on Power Systems 25 (1) (2010) 371-380.

[3] O. van Vliet, A. S. Brouwer, T. Kuramochi, M. van den Broek, A. Faaij, Energy use, cost and CO2 emissions of electric cars, Journal of Power Sources 196 (4) (2011) 2298 - 2310.

[4] W. Kempton, J. Tomic, Vehicle-to-grid power implementation: From stabilizing the grid to supporting large-scale renewable energy, Journal of Power Sources 144 (1) (2005) 280 - 294.

[5] J. García-Villalobos, I. Zamora, J. S. Martín, F. Asensio, V. Aperribay, Plug-in electric vehicles in electric distribution networks: A review of smart charging approaches, Renewable and Sustainable Energy Reviews 38 (2014) $717-731$. 
[6] K. Knezović, M. Marinelli, P. Codani, Y. Perez, Distribution grid services and flexibility provision by electric vehicles: A review of options, in: 2015 50th International Universities Power Engineering Conference (UPEC), 2015.

[7] Smart Energy Demand Coalition (SEDC), Mapping Demand Response in Europe Today (2015).

[8] Council of European Energy Regulators (CEER), Status review on the transposition of unbundling requirements for DSOs and closed distribution system operators (April 2013).

[9] The Union of the Electricity Industry - Eurelectric, Power distribution in Europe (2013).

[10] The Union of the Electricity Industry - Eurelectric, Flexibility and aggregation requirements for their interaction in the market (2014).

[11] The Union of the Electricity Industry - Eurelectric, Network tariff structure for a smart energy system (2013).

[12] J. Hu, H. Morais, T. Sousa, M. Lind, Electric vehicle fleet management in smart grids: A review of services, optimization and control aspects, Renewable and Sustainable Energy Reviews 56 (2016) 1207 $-1226$.

[13] F. Mwasilu, J. J. Justo, E. K. Kim, T. D. Do, J. W. Jung, Electric vehicles and smart grid interaction: A review on vehicle to grid and renewable energy sources integration, Renewable and Sustainable Energy Reviews 34 (2014) $501-516$.

[14] H. L. Ferreira, A. Costescu, A. L'Abbate, P. Minnebo, G. Fulli, Distributed generation and distribution market diversity in europe, Energy Policy 39 (9) (2011) 5561 - 5571.

[15] EvolvDSO consortium, Deliverable 1.2: Evaluation of current market architectures and regulatory frameworks and the role of DSOs (2014).

[16] Danish Standards Foundation, DS/EN 50160:2010 Voltage characteristics of electricity supplied by public electricity networks (Aug 2010).

[17] Green eMotion consortium, Deliverable 4.3 - B2: Grid impact studies of electric vehicles; Reinforcement costs in low-voltage grids (2013).

[18] V. Marques, N. Bento, P. M. Costa, The "smart paradox": Stimulate the deployment of smart grids with effective regulatory instruments, Energy 69 (2014) 96 - 103.

[19] R. J. Bessa, M. A. Matos, Economic and technical management of an aggregation agent for electric vehicles: a literature survey, European Transactions on Electrical Power 22 (3) (2012) 334-350.

[20] T. G. S. Román, I. Momber, M. R. Abbad, A. S. Miralles, Regulatory framework and business models for charging plug-in electric vehicles: Infrastructure, agents, and commercial relationships, Energy Policy 39 (10) (2011) $6360-6375$.

[21] K. Zhou, L. Cai, Randomized PHEV charging under distribution grid constraints, IEEE Transactions on Smart Grid 5 (2) (2014) 879-887. 
[22] N. Leemput, F. Geth, J. Van Roy, A. Delnooz, J. Buscher, J. Driesen, Impact of electric vehicle onboard single-phase charging strategies on a Flemish residential grid, IEEE Transactions on Smart Grid 5 (4) (2014) 1815-1822.

[23] K. Knezović, M. Marinelli, Phase-wise enhanced voltage support from electric vehicles in a danish low-voltage distribution grid, Electric Power Systems Research 140 (2016) $274-283$.

[24] L. Gan, U. Topcu, S. H. Low, Optimal decentralized protocol for electric vehicle charging, IEEE Transactions on Power Systems 28 (2) (2013) 940-951.

[25] L. Zhang, F. Jabbari, T. Brown, S. Samuelsen, Coordinating plug-in electric vehicle charging with electric grid: Valley filling and target load following, Journal of Power Sources 267 (2014) $584-597$.

[26] E. R. Muñoz, G. Razeghi, L. Zhang, F. Jabbari, Electric vehicle charging algorithms for coordination of the grid and distribution transformer levels, Energy 113 (2016) 930 - 942.

[27] E. Sortomme, M. M. Hindi, S. D. J. MacPherson, S. S. Venkata, Coordinated charging of plug-in hybrid electric vehicles to minimize distribution system losses, IEEE Transactions on Smart Grid 2 (1) (2011) $198-205$.

[28] S. Deilami, A. S. Masoum, P. S. Moses, M. A. S. Masoum, Real-time coordination of plug-in electric vehicle charging in smart grids to minimize power losses and improve voltage profile, IEEE Transactions on Smart Grid 2 (3) (2011) 456-467.

[29] C. Eid, L. A. Bollinger, B. Koirala, D. Scholten, E. Facchinetti, J. Lilliestam, R. Hakvoort, Market integration of local energy systems: Is local energy management compatible with european regulation for retail competition?, Energy 114 (2016) 913 - 922.

[30] M. Marinelli, S. Martinenas, K. Knezović, P. B. Andersen, Validating a centralized approach to primary frequency control with series-produced electric vehicles, Journal of Energy Storage 7 (2016) 63 - 73.

[31] S. Martinenas, K. Knezović, M. Marinelli, Management of power quality issues in low voltage networks using electric vehicles: Experimental validation, IEEE Transactions on Power Delivery 32 (2) (2017) 971-979.

[32] K. Knezović, S. Martinenas, P. B. Andersen, A. Zecchino, M. Marinelli, Enhancing the role of electric vehicles in the power grid: Field validation of multiple ancillary services, IEEE Transactions on Transportation Electrification 3 (1) (2017) 201-209.

[33] P. Siano, Demand response and smart grids - a survey, Renewable and Sustainable Energy Reviews 30 (2014) $461-478$.

[34] European Commission, Directive 2009/72/EC of the European Parliament and of the Council of 13 July 2009 concerning common rules for the internal market in electricity and repealing directive 2003/54/EC, Publications Office of the European Union.

[35] European Commission, Commission Recommendation of 9 March 2012 on Preparations for the roll-out 
of smart metergin systems, Publications Office of the European Union.

[36] European Commision, Benchmarking smart metering deployment in the EU-27 with a focus on electricity, Publications Office of the European Union.

[37] C. Cambini, A. Meletiou, E. Bompard, M. Masera, Market and regulatory factors influencing smartgrid investment in europe: Evidence from pilot projects and implications for reform, Utilities Policy 40 (2016) $36-47$.

[38] L. L. Schiavo, M. Delfanti, E. Fumagalli, V. Olivieri, Changing the regulation for regulating the change: Innovation-driven regulatory developments for smart grids, smart metering and e-mobility in Italy, Energy Policy 57 (2013) 506 - 517.

[39] Forum Netztechnik/Netzbetrieb (FNN), VDE-AR-N 4105 Technische Mindestanforderungen an den Anschluss und Betrieb von Erzeugungsanlagen am Niederspannungsnetz [Minimum technical requirements for the connection and operation power generation systems connected to the low-voltage distribution network], Frankfurt, (in German) (2011).

[40] Comitato Elettrotecnico Italiano, CEI 0-21 Regola tecnica di riferimento per la connessione di Utenti attivi e passivi alle reti BT delle imprese distributrici di energia elettrica [Reference technical rules for the connection of active and passive users to LV distribution networks], Milano, (in Italian) (2011).

[41] N. Leemput, F. Geth, J. V. Roy, J. Büscher, J. Driesen, Reactive power support in residential LV distribution grids through electric vehicle charging, Sustainable Energy, Grids and Networks 3 (2015) $24-35$.

[42] International Electrotechnical Commission (IEC) standard, 61851-1:2010 Electric vehicle conductive charging system - Part 1: General requirements.

[43] Society of Automotive Engineers (SAE) standard, J1772:2010 Electric vehicle and plug in hybrid electric vehicle conductive charge coupler.

[44] International Electrotechnical Commission (IEC) standard, 15118-1:2013 Road vehicles - Vehicle to grid communication interface.

[45] Open Charge Alliance, Open charge point protocol (OCPP) 1.6.

[46] e-Laad and Smartlab and Blue Corner, Open clearing house protocol (OCHP) 1.3.

[47] Open Charge Alliance, Open smart charging protocol (OSCP) 1.0.

[48] M. Vallés, J. Reneses, R. Cossent, P. Frías, Regulatory and market barriers to the realization of demand response in electricity distribution networks: A european perspective, Electric Power Systems Research 140 (2016) $689-698$.

[49] T. Gómez, Electricity Distribution, Springer London, London, 2013, pp. 199-250.

[50] J. Katz, Linking meters and markets: Roles and incentives to support a flexible demand side, Utilities Policy 31 (2014) $74-84$. 
[51] Universal Smart Grid Framework, USEF: The framework explained (2015).

[52] S. S. Torbaghan, N. Blaauwbroek, P. Nguyen, M. Gibescu, Local market framework for exploiting flexibility from the end users, 13th International Conference on the European Energy Market (EEM) (2016) 1-6.

[53] EvolvDSO consortium, Deliverable 1.3: Preliminary assessment of the future roles of DSOs, future market architectures and regulatory frameworks for network integration of DRES (2014).

[54] iPower consortium, FLECH - Market specification analysis (2013).

[55] European network of transmission system operators for electricity (ENTSO-E), Towards smarter grids: Developing TSO and DSO roles and interactions for the benefit of consumers (2015).

[56] CEDEC, EDSO, ENTSO-E, Eurelectric, GEODE, General guidelines for reinforcing the cooperation between TSOs and DSOs (2015).

[57] H. Hansen, H. H. Holm-Hansen, O. Samuelsson, L. H. Hansen, H. W. Bindner, H. Jóhannsson, P. Cajar, Coordination of system needs and provision of services, 2013.

[58] E. M. Larsen, Demand response in a market environment, Ph.D. thesis, Technical University of Denmark, Denmark (2016). 